\title{
PART IV RESEARCH
}

Balanced somewhere between thinking and doing is the need for verification by experimentation. We should never move too far away from the spirit of inquiry which drives us to examine the fundamental assumptions of our professions. If our elegant models and programs have no grounding in reality, they will fail us in practice. Therefore, we include a section on research efforts and encourage others to share their findings with our readers.

The two papers in this section include a study sponsored by POD itself on the health of the profession as reflected in the activities of development on various campuses and an analysis of our target population, the faculty, who they are and why they're here. Both papers should result in more questions and encourage more research as well as pointing the way for better practice. 